\title{
Composição de grupos, conflitos intergrupais e uso do espaço em Callithrix penicillata (Geoffroy, 1812) introduzidos em um fragmento urbano
}

\author{
Luciana Zago ${ }^{1,2 *}$ \\ João M. D. Miranda ${ }^{1,2}$ \\ Cristina V. Santos ${ }^{3}$ \\ Fernando C. Passos ${ }^{1}$ \\ Paulo C. A. Simões-Lopes ${ }^{4}$ \\ ${ }^{1}$ Laboratório de Biodiversidade, Conservação e Ecologia de Animais Silvestres \\ Departamento de Zoologia, Setor de Ciências Biológicas, Universidade Federal do Paraná \\ Jardim das Américas, Caixa Postal 19020, CEP 81531-980, Curitiba - PR, Brasil \\ ${ }^{2}$ Laboratório de Biodiversidade do Sul do Brasil \\ Universidade Estadual do Centro-Oeste, Guarapuava - PR, Brasil \\ ${ }^{3}$ Laboratório de Educação Cerebral, Departamento de Psicologia \\ Universidade Federal de Santa Catarina, Florianópolis - SC, Brasil \\ ${ }^{4}$ Departamento de Ecologia e Zoologia, Centro de Ciências Biológicas \\ Universidade Federal de Santa Catarina, Florianópolis - SC, Brasil \\ * Autor correspondente \\ luazagos@gmail.com
}

Submetido em 18/11/2013

Aceito para publicação em 05/03/2014

\section{Resumo}

O uso do espaço pelo gênero Callithrix pode ser relacionado a diferentes fatores. O objetivo deste trabalho foi avaliar as influências de diferentes fatores sobre o uso do espaço em C. penicillata introduzidos em um fragmento urbano. Foram monitorados dois grupos, denominados GL e GG, em duas fases de seis meses no Parque Ecológico do Córrego Grande, Florianópolis, SC. Ambos os grupos eram compostos por oito indivíduos ao início do estudo. Ao longo da Fase I ocorreram desaparecimentos de indivíduos em GL e nascimentos em GG, alterando as composições para cinco e 11 indivíduos, respectivamente. Na Fase II, GL deslocou-se para uma área pouco acessível impossibilitando a realização de observações suficientes. Em GG desapareceram três indivíduos e nasceram outros dois. Comportamentos agonísticos intergrupais foram registrados em todos os meses da Fase I, enquanto na Fase II houve uma abrupta redução. Ocorreram sobreposições das áreas de vida em toda Fase I. Entre a Fase I e II, GL afastou-se da sobreposição e GG ocupou espaços de GL. Tais mudanças parecem associadas ao aumento de indivíduos em GG e sua necessidade em acessar recursos alimentares. As dinâmicas no uso do espaço parecem resultantes de: limitações espaciais, conflitos intergrupais, composições dos grupos e disponibilidade de recursos alimentares.

Palavras-chave: Área de Vida; Comportamentos Agonísticos Intergrupais; Mico-estrela; Recursos Alimentares 


\section{Abstract}

Group composition, intergroup conflicts and use of space in Callithrix penicillata (Geoffroy, 1812) introduced in an urban patch. The use of space by the Callithix genus can be related to different factors. The objective of this study was to evaluate the influences of different factors on the use of space in C. penicillata introduced in an urban patch. Two groups, called GL and GG, were monitored in two six-month phases at Parque Ecológico do Córrego Grande, Florianópolis, SC, Brazil. Both groups consisted of eight individuals at the beginning of the study. Throughout Phase I some GL individuals disappeared and births occurred among GG, changing the groups' composition to five and 11 individuals, respectively. In Phase II, GL moved to an inaccessible area preventing sufficient observations. Three GG individuals disappeared and two others were born. Intergroup agonistic behaviors were recorded in all Phase I months, while an abrupt reduction occurred in Phase II. Home range overlaps occurred throughout Phase I. Between Phases I and II, GL left the overlapping area and GG occupied the GL spaces. These changes seem to be related to the increase in GG individuals and their need to access food resources. The use of space dynamics seems to result from spatial limitations, intergroup conflicts, group compositions and availability of food resources.

Key words: Food Resources; Home Range; Intergroup Agonistic Behaviors; Marmosets

\section{Introdução}

O gênero Callithrix é composto por seis espécies de primatas (RYLANDS; MITTERMEIER, 2009) que se apresentam em estados de conservação distintos (CHIARELLO et al., 2008). Callithrix aurita (Humboldt, 1812) e C. flaviceps (Thomas, 1903) encontram-se ameaçadas de extinção principalmente devido à perda e descaracterização de habitat, além da hibridização e competição com espécies congêneres introduzidas em seus habitats (CHIARELLO et al., 2008). Já $C$. geoffroyi e C. khulii estão fora da lista de espécies ameaçadas, enquanto C. penicillata (É. Geoffroy, 1812) e C. jacchus (Linnaeus, 1758) além de estarem fora da lista de espécies ameaçadas, possuem amplas distribuições geográficas e ocorrem frequentemente em grandes densidades populacionais em ambientes fragmentados e em habitats fora de suas distribuições naturais (FERRARI, 2009).

Tamanhas diferenças nos riscos de extinção das espécies do gênero Callithrix relacionam-se ao estado de conservação de seus respectivos habitats naturais (FERRARI, 2009) e também a adaptações que fazem com que algumas espécies sejam ecologicamente mais flexíveis que outras (RYLANDS, 1996). Todas as espécies desse gênero possuem adaptações morfológicas dentárias e do trato digestivo que os permitem escavar troncos de árvores e consumir a goma que é então exsudada (HERSHKOVITZ, 1977). Entretanto, C. penicillata e $C$. jacchus são espécies com maior especialização dentária para a gomivoria, enquanto $C$. flaviceps e $C$. aurita são espécies com menor especialização para esta atividade alimentar (HERSHKOVITZ, 1977; RYLANDS; FARIA, 1993).

Segundo Burt (1943) a área de vida é a área utilizada por um animal para suas atividades normais a fim de obter alimento, reproduzir e cuidar dos filhotes. Portanto, as áreas de vida de animais ou de grupos de animais devem possuir o tamanho necessário para que com os recursos alimentares existentes possam suprir suas necessidades metabólicas (HARVEY; CLUTTONBROCK, 1981; GRANT et al., 1992). Os exsudatos são ricos em carboidratos, estando disponíveis em todas as estações do ano o que permite que mesmo em épocas de escassez de outros recursos alimentares (e.g. frutos e insetos), as necessidades energéticas desses primatas sejam supridas (FERRARI, 1993). Assim, os grupos de espécies mais gomívoras possuem os menores valores encontrados na literatura para suas áreas de vida anuais, como 0,7ha para C. jacchus (CASTRO, 2003). Já grupos de espécies menos hábeis para a gomivoria necessitam áreas de vida anuais maiores para obter suficiência em recursos alimentares, como 58,3ha para C. kuhlii (RABOY et al., 2008).

Variações intraespecíficas nas áreas de vida nas espécies do gênero Callithrix, por sua vez, podem estar associadas a diferenças na qualidade do habitat, disponibilidade e distribuição das fontes de alimentos e a 
existência de pressões antrópicas, como argumentado por Miranda e Faria (2001) para C. penicillata. Para a mesma espécie, Zago (2012) aponta que além da disponibilidade e qualidade de recursos alimentares, as composições dos grupos podem influenciar a forma como esses animais utilizam o espaço, bem como a forma como defendem esse espaço de grupos vizinhos. O presente trabalho tem como objetivo avaliar as influências que a composição de grupos, os conflitos intergrupais e a disponibilidade de recursos alimentares podem ter sobre e as dinâmicas do uso do espaço de C. penicillata introduzidos em um fragmento urbano.

\section{Material e Métodos}

O estudo foi realizado no Parque Ecológico do Córrego Grande (PECG), um fragmento urbano de 21,5 ha de Floresta Ombrófila Densa em estágios iniciais e médios de regeneração, localizado na Ilha de Santa Catarina $\left(27^{\circ} 35^{\prime} \mathrm{S}, 48^{\circ} 30^{\prime} \mathrm{W}\right)$. Dois grupos de C. penicillata, ambos com oito indivíduos no início do estudo, foram monitorados e identificados pelas siglas GL e GG.

O período de amostragem foi dividido em duas fases: Fase I, de outubro de 2008 a março de 2009, e Fase II, de abril de 2009 a setembro de 2009. Em cada fase foi monitorado um grupo principal com duas amostragens mensais de um dia completo (considerouse um dia completo o período entre a hora em que se encontrou o grupo até a mesma hora do dia seguinte), totalizando dois dias completos mensais, enquanto o outro grupo foi monitorado com amostragens de um dia completo por mês. Na Fase I, o grupo principal foi GL, enquanto na Fase II, devido a mudanças nas áreas de vida de GL que dificultaram suas amostragens, o grupo principal foi GG.

Para ambos os grupos foram registradas suas localizações, através de quadrados de locação de $25 \times 25 \mathrm{~m}$ identificados por um sistema alfanumérico, a cada 10 minutos. Posteriormente, foram calculadas, pelo método do esquadrinhamento (CULLEN JR; VALLADARES-PADUA, 1997), suas áreas de vida acumuladas no período de um ano e em períodos de três meses. Também em períodos de três meses foram calculadas as diferentes proporções do uso dos quadrantes bem como os recursos alimentares utilizados em cada quadrante, a localização das árvores de dormida utilizadas e os quadrantes onde ocorreram encontros intergrupais. Os recursos alimentares utilizados foram categorizados em "presas animais", "frutos", "exsudados" e "alimentos suplementares" (oferecidos por visitantes e vizinhos do PECG). Para o grupo principal (amostrado dois dias por mês) registrou-se também todos os comportamentos agonísticos intergrupais (compostos por vocalizações, pilo-ereção, exibição de genitália, perseguições e até agressões físicas como tapas e mordidas) obtidos pelo método de varredura instantânea com anotação pontual a cada 10 minutos (ALTMANN, 1974).

\section{Resultados}

Os grupos GL e GG eram compostos por oito indivíduos ao início da Fase I. Em GL ocorreu o desaparecimento de um indivíduo em dezembro e de outros dois em fevereiro, permanecendo apenas cinco indivíduos no grupo até o final da Fase I (Tabela 1). Já para GG, ocorreu o nascimento de três infantes em fevereiro, aumentando o grupo para 11 indivíduos até o final da Fase I (Tabela 1).

No início da Fase II, GL permanecia com cinco indivíduos quando passou a viver em uma área alagada composta por vegetação densa. Os esforços de amostragem realizados desde então resultaram apenas em breves encontros com poucos indivíduos do grupo, sendo insuficientes para qualquer inferência. Já para GG, no início da Fase II desapareceram dois indivíduos, com o grupo permanecendo com nove indivíduos até julho, quando desapareceu mais um indivíduo. Ao final de tal fase GG voltou a aumentar para 10 indivíduos com o nascimento de dois infantes (Tabela 1).

Os comportamentos agonísticos intergrupais registrados no presente trabalho ocorreram em todos os meses da Fase I, enquanto na Fase II houve uma abrupta redução nos registros, não ocorrendo em quatro dos seis meses desta fase (Figura 1). 
TABELA 1: Composição dos grupos GL e GG de Callithrix penicillata habitantes do PECG [categorias etárias propostas por Yamamoto (1993)] e número de dias por mês em esforços amostrais no período de outubro de 2008 a setembro de 2009 (células vazias representam meses em que os esforços amostrais foram insuficientes para se determinar as composições dos grupos com exatidão).

\begin{tabular}{|c|c|c|c|c|c|c|c|c|c|c|c|c|}
\hline Mês & Out & Nov & Dez & Jan & Fev & Mar & Abr & Mai & Jun & Jul & Ago & Set \\
\hline GRUPO GL & \multicolumn{6}{|c|}{ Fase I } & \multicolumn{6}{|c|}{ Fase II } \\
\hline Amost. (dias/mês) & 2 & 2 & 2 & 2 & 2 & 2 & 1 & 1 & 1 & 1 & 1 & 1 \\
\hline Adultos & 5 & 5 & 4 & 4 & 4 & 4 & 4 & & & & & \\
\hline Subadultos & 0 & 0 & 0 & 3 & 1 & 1 & 1 & & & & & \\
\hline Juvenis & 3 & 3 & 3 & 0 & 0 & 0 & 0 & & & & & \\
\hline Infantes & 0 & 0 & 0 & 0 & 0 & 0 & 0 & & & & & \\
\hline TOTAL & 8 & 8 & 7 & 7 & 5 & 5 & 5 & & & & & \\
\hline GRUPO GG & \multicolumn{6}{|c|}{ Fase I } & \multicolumn{6}{|c|}{ Fase II } \\
\hline Amost. (dias/mês) & 1 & 1 & 1 & 1 & 1 & 1 & 2 & 2 & 2 & 2 & 2 & 2 \\
\hline Adultos & & & 6 & 6 & 6 & 6 & 5 & 5 & 6 & 5 & 5 & 5 \\
\hline Subadultos & & & 0 & 2 & 2 & 2 & 1 & 1 & 0 & 0 & 0 & 0 \\
\hline Juvenis & & & 2 & 0 & 0 & 0 & 0 & 0 & 0 & 3 & 3 & 3 \\
\hline Infantes & & & 0 & 0 & 3 & 3 & 3 & 3 & 3 & 0 & 0 & 2 \\
\hline TOTAL & & & 8 & 8 & 11 & 11 & 9 & 9 & 9 & 8 & 8 & 10 \\
\hline
\end{tabular}

FIGURA 1: Número de registros de comportamentos agonísticos intergrupais no PECG para os grupos de Callithrix penicillata: GL, na Fase I de outubro de 2008 a março de 2009, e GG, na Fase II de Abril a Setembro de 2009.

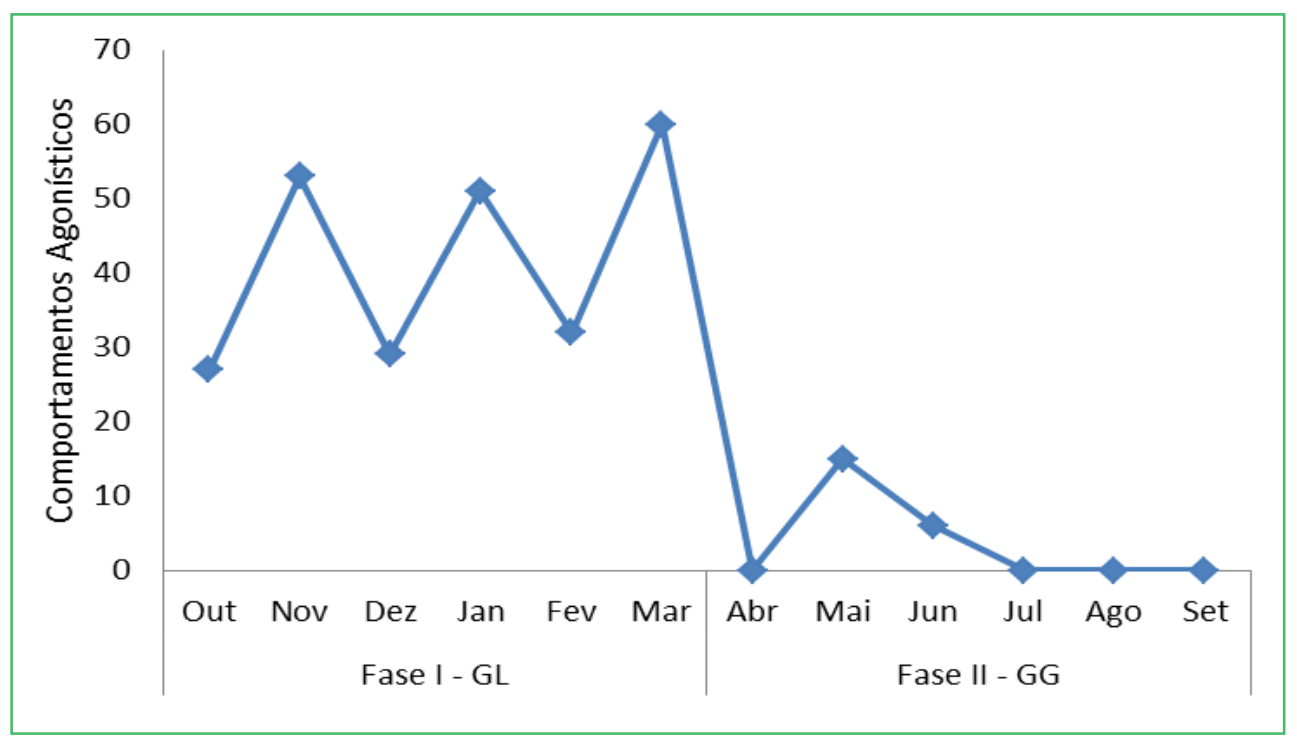


As áreas de vida anuais dos grupos estudados foram de: 3,9 ha para GL e 5 ha para GG e estas sobrepuseram-se em 2,9 ha (Figura 2). Ocorreram encontros intergrupais entre GG e um terceiro grupo denominado GP, os quadrantes onde esses encontros ocorreram foram considerados como sobreposição das áreas de vida destes grupos e somaram 0,4 ha.

O elevado número de comportamentos agonísticos intergrupais registrado na Fase I (Figura 1) coincidiu com grande sobreposição nas áreas de vida de GL e GG e com mudanças nas localizações das mesmas (Figuras 3a e 4a). Os limites da área de GG sobrepuseram-se cada vez mais com a de GL, onde encontros intergrupais ocorreram com grande frequência. A área de GL, por sua vez, foi deslocando-se num sentido oposto a sobreposição com GG e aos encontros intergrupais (Figuras 3a e 4a). Na Fase II (Figuras 5a e 6a), a nova área de GG ocupou espaços antes exclusivos a GL, enquanto este não foi mais avistado nestas localidades. Encontros intergrupais registrados na área de vida de GG também envolveram um terceiro grupo, denominado GP (Figuras 4a e 5a).
FIGURA 2: Tamanho das áreas de vida anuais no PECG, no período de outubro de 2008 a setembro de 2009 , dos grupos de Callithrix penicillata GL e GG, bem como suas sobreposições entre si e com um terceiro grupo, denominado GP.

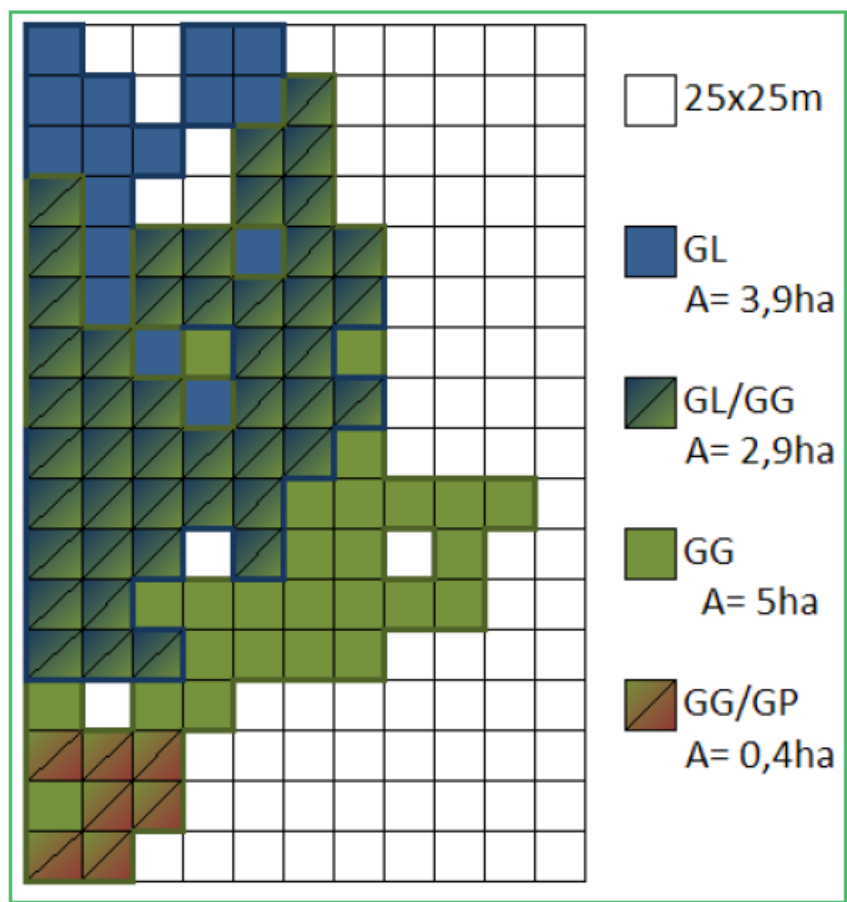

FIGURA 3: (a) Áreas de vida, sobreposições das áreas de vida e quadrantes onde ocorreram encontros intergrupais e árvores de dormida no PECG para os grupos GL e GG, de Callithrix penicillata, no período de outubro a dezembro; (b) porcentagem de uso dos quadrantes e recursos alimentares explorados em cada quadrante utilizado no período de outubro a dezembro pelo grupo GL.

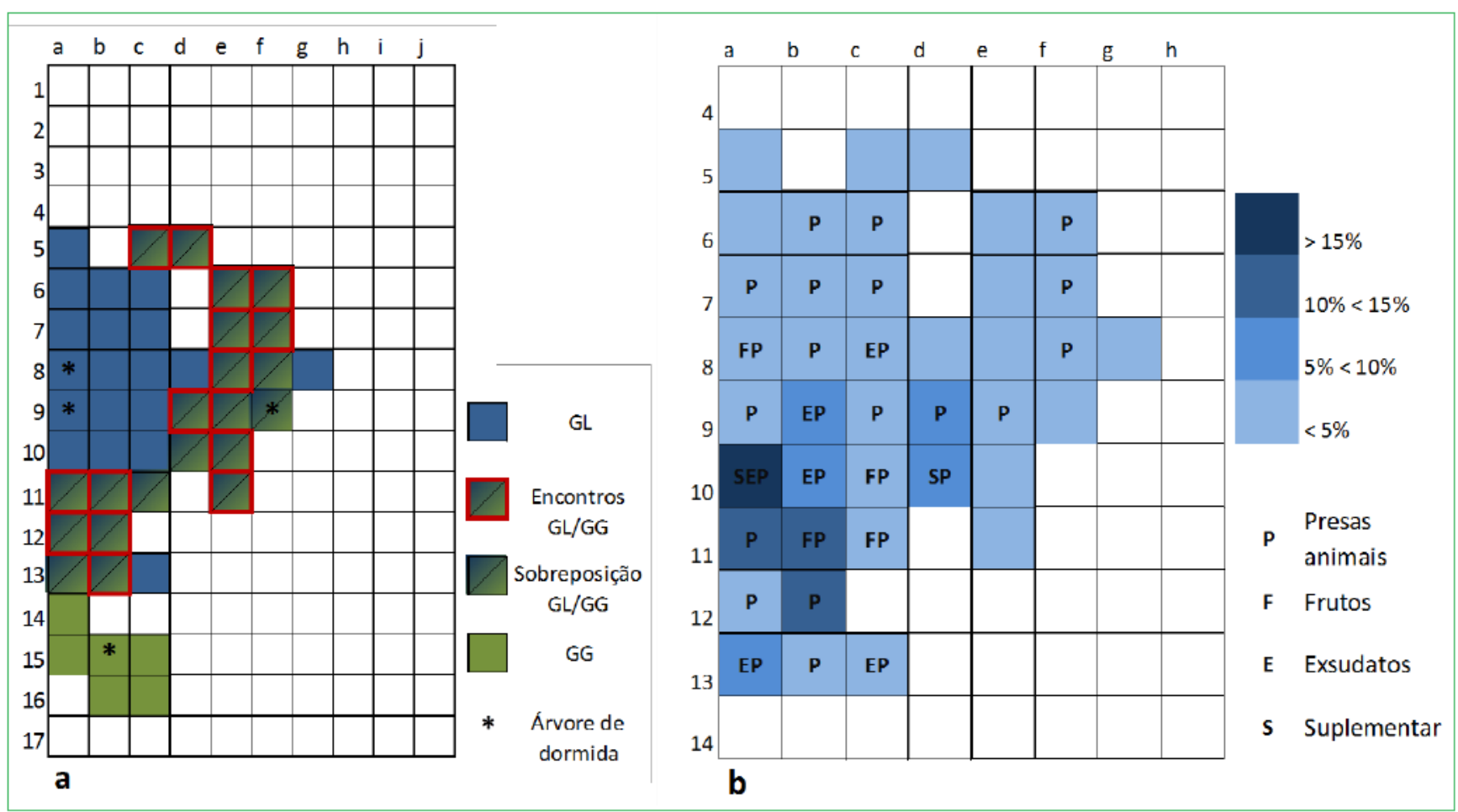


FIGURA 4: (a) Áreas de vida, sobreposições das áreas de vida e quadrantes onde ocorreram encontros intergrupais e árvores de dormida no PECG para os grupos GL, GG e GP de Callithrix penicillata, no período de janeiro a março; (b) porcentagem de uso dos quadrantes e recursos alimentares explorados em cada quadrante utilizado no período de Janeiro a Março pelo grupo GL.

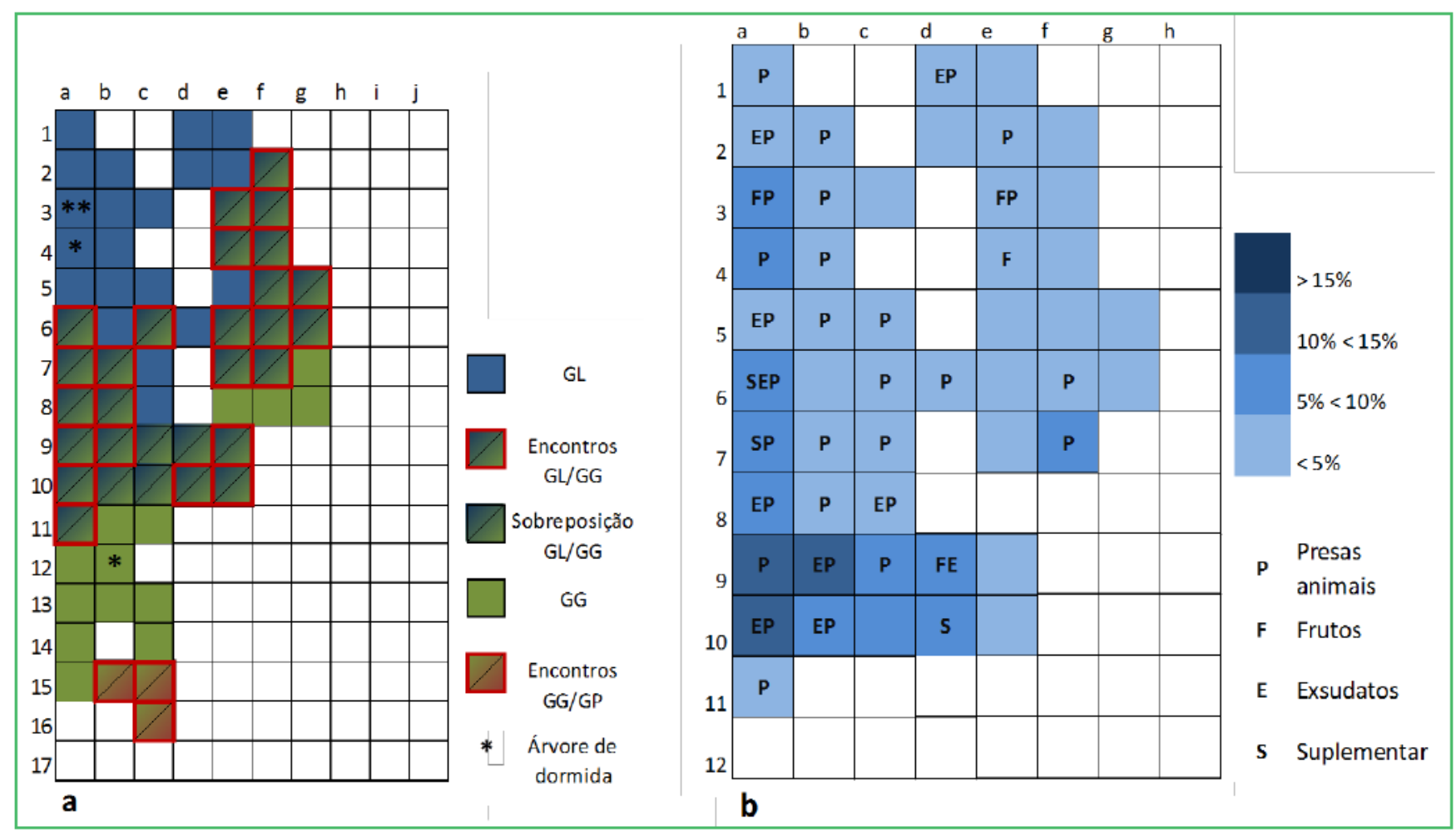

FIGURA 5: (a) Áreas de vida, sobreposições das áreas de vida e quadrantes onde ocorreram encontros intergrupais e árvores de dormida no PECG para o grupo GG, de Callithrix penicillata, no período de abril a junho; (b) porcentagem de uso dos quadrantes e recursos alimentares explorados em cada quadrante utilizado no período de abril a junho pelo grupo GG.

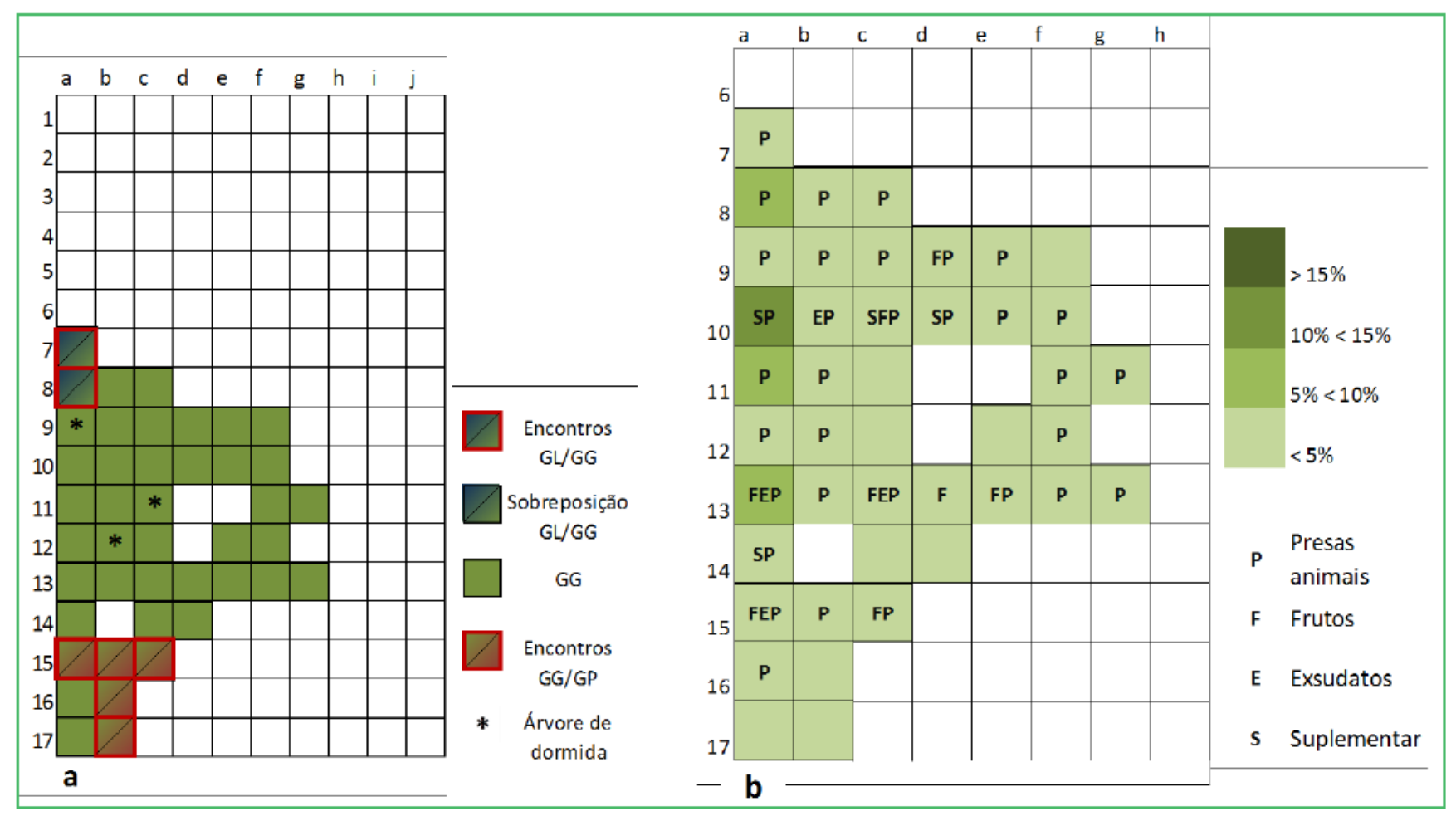


FIGURA 6: (a) Áreas de vida, sobreposições das áreas de vida e quadrantes onde ocorreram encontros intergrupais e árvores de dormida no PECG para o grupo GG, de Callithrix penicillata, no período de julho a setembro; (b) porcentagem de uso dos quadrantes e recursos alimentares explorados em cada quadrante utilizado no período de julho a setembro pelo grupo GG.

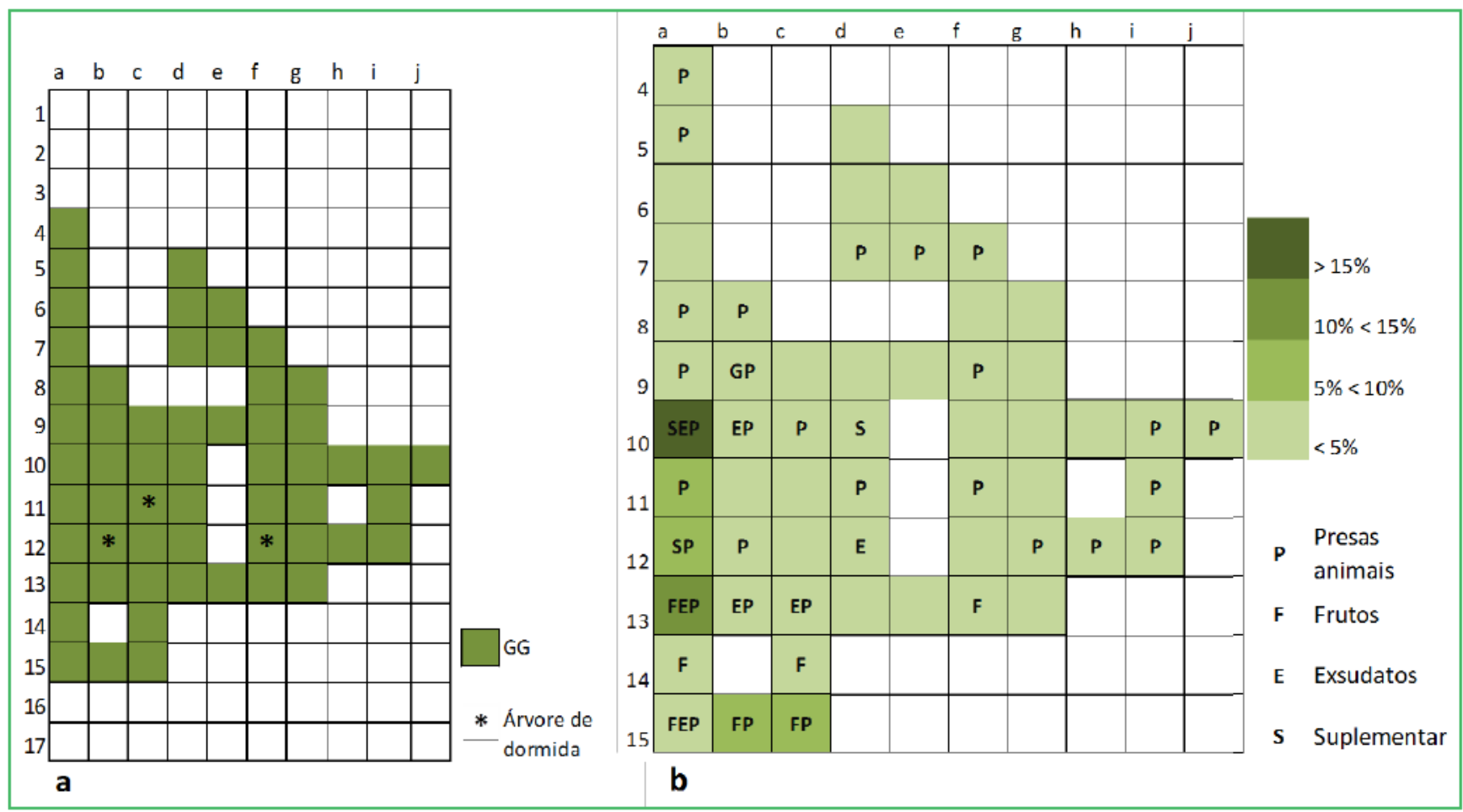

Em ambas as fases os dois grupos utilizaram com maior frequência quadrantes situados próximos ao limite do fragmento florestal (quadrantes da coluna "a", Figuras 3b, 4b, 5b e 6b). Em tais quadrantes ambos os grupos utilizaram árvores para dormida e exploraram árvores de fruto e exsudato, presas animais e alimentos suplementares. O único quadrante utilizado com frequência superior a $15 \%$ (quadrante 10a, Figuras $3 \mathrm{~b}$, $4 \mathrm{~b}, 5 \mathrm{~b}$ e $6 \mathrm{~b}$ ) foi utilizado por ambos os grupos para a obtenção de alimentos suplementares. Estes alimentos foram fornecidos por moradores de uma residência do entorno do PECG através de uma plataforma de alimentação montada no limite do fragmento florestal.

\section{Discussão}

As áreas de vida encontradas neste trabalho, ainda que pequenas, estão dentro dos valores relatados para o gênero Callithrix (RYLANDS; FARIA, 1993; ZAGO 2012). A utilização mais frequente de quadrantes próximos aos limites do PECG (quadrantes da coluna “a” nas Figuras 3 a 6) por ambos os grupos em períodos diferentes pode estar relacionada ao ambiente de borda de floresta. Estes habitats, preferidos por espécies de Callithrix, são caracterizados por uma vegetação densa que auxilia a proteção contra predadores, facilita a movimentação e é abundante em presas animais (RYLANDS, 1996). Lianas e epífitas presentes em abundância nesses hábitats são relatadas como importantes microhabitats para o forrageio de presas por primatas da família Callitrichidae (PASSOS; KEUROGHLIAN, 1999; KEUROGHLIAN; PASSOS, 2001; PASSOS; ALHO, 2001).

Embora o quadrante mais frequentado por ambos os grupos (quadrante 10a nas Figuras 3 a 6) esteja inserido em um ambiente característico de borda de floresta, o uso deste quadrante não está relacionado apenas a estas características, já que há outros quadrantes semelhantes não utilizados frequentemente. $\mathrm{O}$ uso de tal quadrante (quadrante "10a", Figuras 3 a 6) é provavelmente relacionado à disponibilidade frequente de alimentação suplementar oferecida por moradores do entorno do 
PECG, já que permanecendo ali, mesmo realizando outras atividades, os animais podem monitorar a plataforma em que recebem alimentos. Semelhante resultado é relatado por Boere et al. (2000), onde grupos de $C$. penicillata também preferiram o uso de quadrantes com recipientes para alimentação suplementar. Os alimentos suplementares podem possibilitar um grande aporte energético, já que exigem esforços muito menores do que outros recursos alimentares para sua obtenção. A possibilidade desse maior aporte energético pode ser um fator que determine a escolha dos animais em manter-se nesses quadrantes.

Outros quadrantes com grande frequência de uso foram, em sua maioria, utilizados para a exploração de exsudatos, frutos ou árvores de dormida. Tais dados assemelham-se ao encontrado na distribuição natural (RYLANDS; FARIA, 1993; PASSAMANI; RYLANDS, 2000), onde a importância de árvores de exsudato na escolha de uso dos quadrantes só foi superada pela disponibilidade de alimentação suplementar (BOERE et al., 2000) ou pela alta produtividade de frutos ao longo de todo o ano (CASTRO et al., 2000). Como encontrado por Boere et al. (2000), embora possa influenciar no uso do espaço, as árvores de dormida parecem ser um fator de importância secundária, sendo superado pela disponibilidade dos recursos exsudato e fruto.

A disponibilidade de recursos alimentares pode também estar relacionada às mudanças nas localizações das áreas de vida dos grupos estudados. O grupo GG permanecia com sua área delimitada pelo perímetro do PECG e pelas áreas de vida dos grupos GP e GL, quando, em fevereiro, nasceram três infantes. Enquanto ocorreu este aumento no número de indivíduos em GG, em GL houve o desaparecimento de indivíduos, de forma que em apenas um mês as diferenças numéricas destes grupos mudaram de um para seis indivíduos. Essa superioridade numérica de GG foi sucedida pelo aumento de encontros intergrupais em março, pela ocupação de GG e desocupação de GL de uma área abundante em frutos, exsudatos e com frequente alimentação suplementar fornecida por moradores do entorno do PECG.

As novas disposições das áreas de vida de GG parecem mais estáveis na Fase II, quando os encontros intergrupais foram reduzidos, chegando a não ocorrer nos últimos três meses. Assim, a mudança na localização da área de vida de GL, cujos espaços eram de seu uso exclusivo e passaram a ser ocupados por GG, parece resultante da necessidade de GG em aumentar seu acesso aos recursos alimentares. Tal suposição é suportada pela observação de que ambos os grupos utilizaram com maior frequência o mesmo quadrante onde era disponibilizada alimentação suplementar.

Segundo Passamani e Rylands (2000) áreas de vida podem estar relacionadas tanto com disponibilidade de recursos, quanto com interações intergrupais. Os resultados encontrados corroboram com tal afirmação, sendo que os tamanhos das áreas de vida e as dinâmicas no uso do espaço encontradas parecem ser resultantes de um conjunto de fatores dados: pela limitação espacial do fragmento florestal, pela disponibilidade de recursos alimentares e pelas composições dos grupos e conflitos intergrupais.

\section{Agradecimentos}

Agradecemos À Universidade Federal de Santa Catarina pela bolsa fornecida a Luciana Zago para a realização deste trabalho, à administração do Parque Ecológico do Córrego Grande e à Fundação Municipal do Meio Ambiente (FLORAM) por permitirem a coleta de dados e ao Conselho Nacional de Desenvolvimento Científico e Tecnológico (CNPq) pela bolsa de produtividade a Fernando C. Passos (300466/2009-9) e Paulo César Simões-Lopes (310796/2009-1).

\section{Referências}

ALTMANN, J. Observational study of behavior: sampling methods. Behaviour, Groningen, v. 40, p. 227-267, 1974.

BOERE, V.; TILLMANN, L.; RESENDE, M. C.; TOMAZ, C. Uso do espaço e comportamento social em sagüis do cerrado (Callithrix penicillata) selvagens, no Centro de Primatologia da Universidade de Brasília. In: ALONSO, C.; LANGGUTH, A. (Ed.). A Primatologia no Brasil 7. Natal/João Pessoa: Editora da UFRN/Editora Universitária, 2000. p. 35-48.

BURT, W. H. Territoriality and home range concepts as applied to mammals. Journal of Mammalogy, Lawrence, v. 24, n. 3, p. 346$352,1943$.

CASTRO, C. S. S. Tamanho da área de vida e padrão de uso do espaço em grupos de sagüis, Callithrix jacchus (Linnaeus) 
(Primates, Callitrichidae). Revista Brasileira de Zoologia, Curitiba, v. 20, n. 1, p. 91-96, 2003.

CAStro, C. S. S.; ARAÚJO, A.; AlHO, C.; FilHO, M. D. Influência da distribuição e disponibilidade dos frutos, na dieta e uso do espaço em Sagüis-do-Nordeste (Callithrix jacchus). In: AlONSO, C.; LANGGUTH, A. (Ed.). A Primatologia no Brasil 7. Natal/João Pessoa: Editora da UFRN/Editora Universitária, 2000. p. 65-80.

CHIAREllO, A. G.; AGUIAR, L. M. S.; CERQUIERA, R.; MELLO, F. R.; RODRIGUES, F. H. G.; SILVA, V. M. F. Mamíferos ameaçados de extinção no Brasil. In: MACHADO, A.; DRUMMOND, G. M.; PAGLIA, A. P. (Ed.). Livro vermelho da fauna brasileira ameaçada de extinção. Belo Horizonte: FNMA/ Fundação Biodiversitas, 2008. p. 681-874.

CULLEN JR., L.; VALLADARES-PADUA, C. Métodos para estudos de ecologia, manejo e conservação de primatas na natureza. In: VALLADARES-PADUA, C., BODMER, R.E.; CULLEN JR., L. (Eds). Manejo e conservação da vida silvestre no Brasil. Belém, Sociedade Civil Mamirauá/CNPq, 1997. p. 239-269.

FERRARI, S. F. Ecological differentiation in the Callitrichidae. In: RYLANDS, A. (Ed.). Marmosets and Tamarins: systematics, behaviour, and ecology. Oxford: Oxford University Press, 1993. p. 315-328

FERRARI, S. F. Conservation of the Marmosets and Callimicos. In: FORD, S. M.; PORTER, L. M.; DAVIS, L. C. (Ed.). The smallest anthropoids: the Marmoset/Callimico radiation. Series Developments in Primatology: Progress and Prospects. New York: Springer Press, 2009. p. 465-477.

GRANT, J. W. A.; CHAPMAN, C. A.; RICHARDSON, K. S. Defended versus undefended home range size of carnivores, ungulates and primates. Behavioral Ecology and Sociobiology, Göttingen, v. 31, p. 149-161, 1992.

HARVEY, P. H.; CLUTTON-BROCK, T. H. Primate home-range size and metabolic needs. Behavioral Ecology and Sociobiology, Göttingen, v. 8, p. 151-155, 1981.

HESHKOVITZ, P. Living new world monkeys (Platyrrhini), with an introduction to primates. Vol. 1. Chicago: The University of Chicago Press. 1977. 1132 p.

KEUROGHLIAN, A.; PASSOS, F. C. Prey foraging behavior, seasonality and time-budgets in black lion tamarins, Leontopithecus chrysopygus (Mikan 1823) (Mammalia, Callitrichidae). Brazilian Journal of Biology, São Carlos, v. 61, n. 3, p. 455-459, 2001.
MIRANDA, G. H. B.; FARIA, D. S. Ecological aspects of blackpincelled Marmoset (Callithrix penicillata) in the Cerradão and dense Cerrado of the Brazilian Central Plateau. Brazilian Journal of Biology, São Carlos, v. 61, n. 3, p. 397-404, 2001.

PASSAMANI, M.; RYLANDS, A. B. Home range of a Geoffroy's marmoset group, Callithrix geoffroyi (Primates, Callithrichidae) in South-Eastern Brazil. Revista Brasileira de Biologia, São Carlos, v. 60, n. 2 , p. $275-281,2000$.

PASSOS, F. C.; ALHO, C. Importância de diferentes microhabitats no comportamento de forrageio por presas do mico-leão-preto, Leontopithecus chrysopygus (Mikan) (Mammalia, Callitrichidae). Revista Brasileira de Zoologia, Curitiba, v. 18, n. 1, p. 335-342, 2001.

PASSOS, F. C.; KEUROGHLIAN, A. Foraging behavior and microhabitats used by black lion tamarins, Leontopithecus chrysopygus (Mikan) (Primates, Callitrichidae). Revista Brasileira de Zoologia, Curitiba, v. 16, suppl. 2, p. 219-222, 1999.

RABOY, B. E.; CANALE, G. R.; DIETZ, J. M. Ecology of Callithrix kuhlii and a Review of Eastern Brazilian Marmosets. International Journal of Primatology, Durham, v. 29, p. 449467, 2008.

RYLANDS, A. B. Habitat and the evolution of social and reproductive behavior in Callitrichidae. American Journal of Primatology, New York, v. 38, p. 5-18, 1996.

RYLANDS, A. B.; FARIA, D. S. Habitats, feeding ecology, and home range size in the genus Callithrix. In: RYLANDS, A. (Ed). Marmosets and Tamarins: systematics, behaviour, and ecology. Oxford: Oxford University Press, 1993. p. 263-272.

RYLANDS, A. B.; MITTERMEIER, R. A. The diversity of the New World Primates (Platyrrhini): an annotated taxonomy. In: GARBER, P. A.; ESTRADA, A.; BICCA-MARQUES, J. C.; HEYMANN, E. W.; STRIER, K. B. (Ed.). South American Primates: comparative perspectives in the study of bahavior, ecology, and conservation. Series Developments in Primatology: Progress and Prospects. New York: Springer Press, 2009. p. 23-54.

YAMAMOTO, M. E. From dependence to sexual maturity: the behavioural ontogeny of Callitrichidae. In: RYLANDS, A. (Ed.). Marmosets and Tamarins: systematics, behaviour, and ecology. Oxford: Oxford University Press, 1993. p. 235- 254.

ZAGO, L. Fatores determinantes no uso do espaço por Callithrix penicillata (E. Geoffroy, 1812) introduzidos em fragmento urbano. 2012. 80 f. Dissertação (Mestrado em Zoologia) Universidade Federal do Paraná, Curitiba. 2012. 